\title{
DIAGNÓSTICO DE CÂNCER DE MAMA: SENTIMENTOS, COMPORTAMENTOS E EXPECTATIVAS DE MULHERES
}

\author{
DIAGNOSIS OF BREAST CANCER: FEELINGS, BEHAVIORS AND EXPECTATIONS OS WOMEN \\ EL DIAGNÓSTICO DEL CANCER DE MAMA: LOS SENTIMENTOS, LAS CONDUCTAS LAS EXPECTATIVAS DE \\ LAS MUJERES
}

\section{Malena de Fátima Silva Regis ${ }^{1}$ Sonia Mara Faria Simões ${ }^{2}$}

\begin{abstract}
RESUMO: O câncer de mama é a neoplasia maligna responsável pelo maior número de óbitos em mulheres no mundo, inclusive no Brasil. Considerando que a mulher vivencia situações singulares, após a descoberta da doença, que interessam à assistência de saúde. Este estudo teve como objetivo identificar os sentimentos, comportamentos e expectativas de mulheres frente ao câncer de mama por meio da revisão sistemática de literatura. Foram identificados onze artigos através dos bancos de dados LILACS e BDENF por meio dos descritores câncer de mama e diagnóstico (de câncer de mama) considerando um período de dez anos. A análise dos artigos demonstrou sentimentos e comportamentos tais como: medo, negação, aceitação da doença, buscando a causa e o constrangimento. Como expectativas, foi possível encontrar uma nova visão da vida após o câncer. Para uma abordagem humanizada a cliente com diagnóstico de câncer de mama faz-se oportuno considerar as emoções desencadeadas com a confirmação do diagnóstico, quase sempre associado à morte, ao medo e ao desespero. Entender as questões referentes ao ajustamento psicossocial envolvidos no câncer de mama, permite ao profissional de saúde, especialmente a enfermagem, avaliar e assistir a mulher de uma forma adequada e singularizada.
\end{abstract}

PALAVRAS-CHAVE: Neoplasias Mamárias; Cuidado em Enfermagem; Diagnóstico.

ABSTRACT: The breast cancer is the responsible malign neoplasm for the largest deaths number in women in the whole world as in Brazil. Considering that woman lives singular situations, after discovering the disease, that is interested area for health care assistance. This study aims to identify the feelings behaviors and expectations of women front of the breast cancer by a systematic review of the literature. It was identified eleven articles in LILACS and BDENF library by means breast cancer and diagnosis (of breast cancer) descriptors, published in last ten years. The article analysis demonstrated feelings and behaviors as fear, denial, disease acceptance, seeking for the cause and the constraint. As expectations it was possible to find a new life perception after the cancer. For an humanistic approach the client with breast cancer diagnosis it is opportune to consider the emotions unchained from the confirmation of the diagnosis, almost always associated to the death, to the fear and the despair. Understanding the subjects concerning to the psycho-social adjustment involved on the breast cancer theme, it allows to the health professional, specifically Nurses, to evaluate and to attend the woman in an appropriate way.

KEYWORDS: Breast cancer; Nursing Care; Diagnosis.

RESUMEN: El cáncer de las mamas es la neoplasia maligna responsable por el mayor número de óbitos en mujeres en el mundo, inclusive en Brasil. Considerando que la mujer vivencia situaciones singulares, después de descubrir la enfermedad, que muy interesan a la asistencia a la salud. O objetivo de esto estudio fue identificar los sentimientos, comportamientos y las expectativas de mujeres delante el cáncer de las mamas por medio de una revisión sistemática de la literatura científica. Fueron identificados once artículos en las bases de datos bibliográficos LILAS y BDENF utilizándose descriptores neoplasmas de la mama y diagnostico (de neoplasmas de la mama), publicados en los últimos diez años. La análisis de los artículos demostró sentimientos e comportamientos tales como el miedo, la negación, aceptación de la enfermedad, buscando la causa y el constreñimiento. Como expectativas, fue posible encontrar una nueva opinión da vida después la descubierta del cáncer. Para una abordaje humanista las clientes con diagnostico de neoplasia de la mama es oportuno considerar las emociones desencadenadas con la confirmación del diagnostico, casi siempre asociado a la muerte, al miedo e al desespero. Entender los temas que tratan de ajustamiento psico-social inherentes en la neoplasia de las mamas, permiten a los profesionales de la salud, especialmente la enfermería, evaluar y atender la mujer de una manera más apropiada.

PALABRAS-CLAVE: Neoplasmas de la mama, Atención de Enfermería; Diagnóstico.

\section{CONSIDERAÇÕES INICIAIS}

Uma das questões de grande relevância na área da Saúde da Mulher é a que se refere à prevenção do câncer de mama, visto ser a maior causa de óbitos por câncer na população feminina, principalmente na faixa etária entre os 40 e 69 anos. De acordo com a dados apresentados pelo Ministério da Saúde com base na Organização Mundial de Saúde (BRASIL, 2003), registrou-se nas décadas de 60 e 70 um aumento de 10 vezes em suas taxas de incidência. 
Segundo o Ministério da Saúde (BRASIL, 2003), no Brasil, o câncer de mama é o que mais causa morte entre as mulheres. Em 2000, foram registradas 8.390 mortes decorrentes deste tipo de câncer. Dos 402.190 novos casos de câncer com previsão de serem diagnosticados em 2003, o câncer de mama será o segundo mais incidente entre a população feminina, sendo o responsável por 41.610 novos casos e 9.335 óbitos.

Ainda de acordo com o Ministério da Saúde (BRASIL, 2004), o objetivo específico para reduzir a mortalidade por câncer de mama na população feminina para os anos de 2004-2007 tem como metas aumentar em $45 \%$ a realização de cirurgias para reconstrução que já está prevista através de uma lei federal, apoiar a capacitação de profissionais da rede na detecção precoce e no tratamento do câncer de mama e elaborar documento de consenso sobre câncer de mama.

Embora o prognóstico para o câncer de mama esteja mudando devido a avanços no diagnóstico e tratamento, as respostas das mulheres à possível doença incluem o medo da desfiguração, perda da atividade sexual e medo da morte.

A escolha em desenvolver este tema como Trabalho Final de Curso de Graduação surgiu após um estudo de caso apresentado por colegas de turma na disciplina Saúde Integral do Adulto e do Idoso II durante o estágio no Hospital Universitário Antonio Pedro. Esse estudo tratava de uma paciente com câncer do colo do útero que sofreria uma histerectomia. Segundo relatos, a paciente estava com muito medo e dúvidas, levantando até a hipótese de "deixar de ser mulher" após a cirurgia. Isto me despertou o interesse em pesquisar os sentimentos e expectativas da mulher na descoberta do diagnóstico de câncer de mama, visto que, como mulheres que somos, valorizamos e atribuímos muito significado ao seio.

Assim, este estudo teve como objetivo identificar os sentimentos/comportamentos e expectativas da mulher na descoberta do diagnóstico de câncer de mama. Para isso, foi elaborada a seguinte questão norteadora: Quais são os sentimentos/comportamentos e expectativas da mulher frente ao diagnóstico de câncer de mama?

Isto porque a palavra câncer traz um estigma muito forte, pois as pessoas logo o associam com a morte. O câncer de mama é ainda mais temido pelo fato de acometer uma parte valorizada do corpo da mulher e que em muitas culturas desempenha uma função significativa da sexualidade da mulher e sua identidade.

Para NEGRINI (1994) a mulher com câncer de mama torna-se uma pessoa duramente atingida física, psicológica e socialmente, tanto pela doença como pelo tratamento. Aceitar sua nova condição, adaptarse a nova imagem de seu corpo exige um esforço muito grande para o qual, não estão preparadas.

Nesse momento de sua de vida, surgem dificuldades que abalam seu equilíbrio e afetam seu relacionamento. A relação enfermeiro-paciente pode desempenhar papel de ajuda, já que a humanização da assistência de enfermagem não vê mais "um órgão doente" e sim a paciente como um todo, com sua história, medos e angústias.

Torna-se importante compreender como uma mulher com diagnóstico de câncer de mama reage frente esta etapa de sua vida, acreditando assim ser oportuno o entrosamento maior entre ela e a equipe. (TRAVELBEE, 1979, apud GRACIOLI et al, 2003) percebe a enfermagem como um processo interpessoal entre dois seres humanos, no qual um deles precisa de ajuda e o outro fornecesse ajuda, sendo motivados a procurar e compreender todas as experiências de vidas ambos.

\section{METODOLOGIA}

Trata-se de um estudo no qual foi utilizada uma revisão sistemática da literatura sendo possível identificar o conhecimento sobre os sentimentos/comportamentos e expectativas de mulheres frente ao câncer de mama.

Em 1995, um grupo de cientistas reunidos em Potsdam (Alemanha) "definiu como revisão sistemática a aplicação de estratégia científicas que limitem o viés de seleção de artigos, avaliem com espírito crítico os artigos e sintetizem todos os estudos relevantes em um tópico específico". (PERISSÉ, 2001, apud GALVÃO et al, 2004)

Segundo LIMA (2000), apud GALVÃO et al (2004) "a revisão sistemática é uma forma de síntese das informações disponíveis em dado momento, sobre um problema específico, de forma objetiva e reproduzível, por meio de método científico"

A utilização estratégica para a busca dos estudos consiste na procura em bases eletrônicas de dados, na busca manual em periódicos, as referências listadas nos estudos identificados e o encontro de material não publicado.

POLIT \& HUNGLER (1995) e GIL (1999) ressaltam que a revisão sistemática consiste num resumo crítico de pesquisa sobre tópico de interesse, geralmente preparado para colocar um problema de pesquisa num contexto, ou para identificar as falhas em estudos anteriores, de modo a justificar uma nova investigação.

As publicações sobre o assunto, contido em periódicos nacionais indexados e não indexados, constituem o objeto desta análise.

Utilizamos duas bases de dados para a realização deste levantamento bibliográfico, a saber: LILACS (Literatura Latino Americano em Ciências de Saúde) BDENF (Base de Dados de Enfermagem).

Para acesso, utilizamos as palavras-chave "câncer de mama", "diagnóstico de câncer de mama".

A população do estudo foi composta por artigos indexados, de acordo com levantamento do banco de dados.

Adotamos como critérios de inclusão das publicações na consulta ao banco de dados referidos.

- Publicação em periódicos nacionais tais como: Revistas Latino-Americana Enfermagem, Brasileira de Cancerologia, Gaúcha de 
Enfermagem, Texto \& Contexto Enfermagem, Acta Paulista de Enfermagem, Brasileira de Enfermagem, Mineira de Enfermagem.

- Pesquisa dos periódicos disponíveis nas Bibliotecas de Enfermagem da Universidade Federal do Rio de Janeiro e Universidade Federal Fluminense.

- Publicações que apresentassem como população de estudo mulheres com diagnóstico de câncer de mama.

As publicações foram numeradas de forma aleatória. Antecedeu a análise, a leitura de todos os textos. A análise das publicações foi desenvolvida e orientada visando à identificação dos sentimentos/comportamentos e expectativas propostas no estudo. (A letra $\mathrm{P}$ - maiúsculo - seguida do número representa a publicação em análise).

\section{RESULTADOS E DISCUSSÃO}

Inicialmente apresentamos o ano de publicação, bem como as fontes das publicações que preencheram os critérios de inclusão deste estudo.

Tabela 1: Fonte de dados

\begin{tabular}{|l|l|l|l|}
\hline $\begin{array}{l}\text { ANO DA } \\
\text { PUBLICAÇÃO }\end{array}$ & \multicolumn{4}{l|}{ FONTE DE DADOS } \\
\hline & LILACS & BDENF & $\begin{array}{l}\text { NÃO } \\
\text { INDEXADOS }\end{array}$ \\
\hline 1993 & & & 1 \\
\hline 1997 & & 2 & \\
\hline 1998 & 1 & & \\
\hline 2001 & 1 & 1 & 1 \\
\hline 2002 & 1 & 1 & \\
\hline 2003 & & & 1 \\
\hline 2004 & & & 1 \\
\hline
\end{tabular}

A amostra das publicações (11) revelou que três estavam indexadas no LILACS e quatro no BDENF. Os demais, quatro publicações, foram encontrados através da busca manual.

A análise será apresentada conforme agrupamento das publicações selecionadas considerando como relevante os sentimentos/comportamentos e expectativas de mulheres frente ao câncer de mama.

Patologias que abalam a estrutura orgânica emocional da mulher, como o câncer de mama, desencadeiam emoções que as deixam fragilizadas, a confirmação de que têm um câncer é sempre associada à morte e assim começam a viver sob o estresse do medo e do desespero. Nesse sentido, ela necessita de um tempo para poder refletir sobre o fato de estar com câncer, mesmo sabendo que terá um futuro incerto, cheio de sentimentos como medo, angústia, dor e sofrimento.

Considerando os temas desenvolvidos nas publicações analisadas evidenciamos como sentimentos/comportamentos: medo, aceitação da doença, negação, buscando a causa e constrangimento e em relação a expectativas: uma nova visão.

\section{SENTIMENTOS/COMPORTAMENTOS MULHERES}

DAS

\section{Medo}

Sabe-se que ainda não é possível tornar realidade a prevenção primária para o câncer de mama, ou seja, impedir que a doença se manifeste. Falar em prevenção do câncer significa falar na detecção precoce, através do auto-exame das mamas. Segundo o Ministério da Saúde (BRASIL, 2004), cerca de $80 \%$ dos tumores são descobertos pela própria mulher, palpando suas mamas incidentalmente.

Quando a mulher realiza um auto-exame e detecta algum nódulo ou alguma alteração o sentimento de medo torna-se presente. Ela toma consciência de dois aspectos simultâneos: pode não ser nada grave ou significar um câncer. Normalmente, ela pensa no pior. (CORBELLINI, 2001)

Segundo FERREIRA (1993) medo é o sentimento de viva inquietação ante a noção do perigo real ou imaginário, de ameaça; pavor; temor; receio.

A descoberta desencadeia de imediato uma reação emocional intensa. Na concepção de algumas pacientes, câncer é sinônimo de morte e a descoberta aconteceu de forma inesperada. (FIALHO \& SILVA, 1993)

O medo com o inesperado são algumas das condições que acabam ocasionando a perda do equilíbrio, da saúde e do self. Para algumas mulheres o câncer de mama é tão temido que elas descrevem a doença com outros nomes.(FUNGHETTO et al, 2003)

Mulheres com câncer de mama denotam o sofrimento que a dor e a doença Ihes trazem, entendemos que para elas o medo da morte faz com que a possibilidade da dor seja muito mais valorizada. (ALMEIDA et al, 2001)

Por estar vivenciando, um mundo desconhecido frente ao câncer de mama e suas repercussões, essas mulheres desencadeiam sensações conflitivas a aflitivas, ocasionando um comportamento de angústia, agitação e medo. (BITTENCOURT \& CADETE, 2002a)

A representação do câncer expressa uma visão assustadora e temerosa, identificada pelas mulheres, 
demonstrando o temor que ele representa em suas vidas. Conforme SILVA \& MAMEDE (1998) o medo está em todas as fases percorridas pela mulher no processo de adoecer. O medo do diagnóstico de câncer torna-se ameaçador, originando, assim reações emocionais que provocarão mudanças no âmbito biológico, mental e social.

\section{Aceitação da doença}

Aceitação para FERREIRA (1993) é consentir em receber, concordar com; aceitação. Algumas mulheres, frente ao de câncer de mama, podem apresentar um comportamento contraditório em relação a demais diante de tal situação.

Para umas um alívio, pois a partir do diagnóstico, poderão iniciar o tratamento e retornar as suas vidas normais. (CORBELLINI, 2001)

Uma das primeiras reações ao perceber algo de "estranho" no seio, é à procura imediata de um médico. (BACKES, 1997)

\section{Negação}

A negação pode ser manifestada como uma forma de "defesa aparente", empregada por muito de nós, quando nos deparamos em situações de dificuldade. Na mulher com câncer de mama não poderia ser diferente, este sentimento surge com intensidade, visto que, ela se encontra vulnerável.

A negação é um mecanismo de defesa muito utilizada em doenças crônicas e, principalmente naquelas estigmatizantes e com forte caráter de desesperança, como é o caso do câncer e esteve na experiência de algumas mulheres que mesmo submetendo-se ao tratamento cirúrgico e/ou tratamentos complementares evitavam um confrontamento com a realidade da doença. (CALIRI et al, 1998)

Para muitas o diagnóstico vem como um choque completo, talvez por não terem noção de que o nódulo poderia ser algo sério ou porque simplesmente usaram o sentimento de negação para anular os sintomas presentes. Muitas vezes a mulher procura protelar o tratamento, devido a causa múltiplas, dentre elas a negação de estar com uma doença grave, fator socioeconômico e fatores psicológicos (CORBELLINI, 2001)

A negação é como uma defesa aparente pode ser manifestada pelo medo de enfrentar a doença e até pelo medo da morte. As doenças de difícil diagnóstico ou que podem deixar marcas físicas no corpo humano possuem uma capacidade de reprimir as emoções e negar os conflitos gerados pela própria doença. (GRACIOLI et al, 2003)

\section{Buscando a causa}

Diante da situação vivenciada, algumas mulheres procuram buscar o entendimento para o surgimento da doença, e muitas vezes, questionam e refletem o
Nesse sentido, a mulher reconhece a necessidade de aceitar sua nova condição de portadora de câncer de mama, e a reorganização de papéis decorrentes dessa experiência, submetendo-se assim ao tratamento, pois deseja alcançar a cura. (BERGAMASCO \& ÂNGELO, 2001)

A aceitação da doença torna-se mais fácil quando as pacientes comparam se com outras mulheres em piores situações ou quando minimizam as conseqüências do tratamento. (CALIRI et al, 1998)

Dessa forma, as mulheres, com muita determinação, demonstravam fé incondicional e submissão à vontade divina, cabendo-lhes, assim, a aceitação e a resignação. (BITTENCOURT \& CADETE, 2002a)

SILVA \& MAMEDE (1998) dizem que a aceitação da situação na qual a mulher se encontra pode ser aparente, momentânea, pois ela se encontra em uma fase inicial e progressiva da doença. Ainda para este autor a aceitação também funciona no sentido de não ter outro jeito como se não houvesse o que fazer, mas permanecendo a expectativa projetada.

porquê de sua doença. Desta forma, buscam em seus comportamentos no passado uma explicação.

Um outro ponto importante surgido foi à necessidade que a maioria das mulheres manifestava em compreender o que estava acontecendo com elas. (BITTENCOURT \& CADETE, 2002a)

Toda pessoa, ao longo de sua vida, depara-se com uma infinidade de situações problemáticas que podem contemplar desde grandes crises, tais como uma doença grave e suas conseqüências. A repressão de sentimentos está ligada ao fato de que, se a pessoa ignorar as emoções como a raiva, ou a ansiedade, então elas simplesmente irão desaparecer

Desgosto e sofrimentos reprimidos ao logo da vida estão associados a fortes sentimentos de culpa. (FERNANDES \& MAMEDE, 2004)

O câncer de mama traz à consciência das mulheres o quanto o cuidado consigo mesma é colocado em segundo plano. Diante da situação vivida, muitas passam a refletir acerca das ações tomadas com relação à sua saúde e percebem o descaso que tiveram com o próprio corpo. (ALMEIDA et al, 2001)

Como causa do processo de adoecimento, mulheres encontram em momentos de grande fragilidade emocional, de conflitos pessoais e familiares de perdas de entes queridos. Uma mulher explicita claramente que sua doença teve origem emocional, a outra atribui a doença uma "pancada" e corte no seio. (BACKES, 1997)

Encontrar uma causa para doença pode ter importante implicação, para mulheres com câncer de mama. Essa busca de significado pode torná-las aptas a conhecer melhor sua situação. Dessa forma, a atribuição causal pode ser importante porque, freqüentemente determina os tipos de estratégias adotadas, pelas mulheres para seu ajustamento e alterações de seu estilo de vida.

\section{Constrangimento}


A palavra câncer traz um estigma muito forte. $\mathrm{O}$ fato de estar com esta doença é para mulher um grande constrangimento, pois as repercussões decorrentes do tratamento abalam a imagem corporal repercutindo assim no seu convívio social.

Algumas mulheres verbalizam mudanças percebidas em estrutura e/ou função do corpo. O não olhar para uma parte do corpo, não tocar, esconder provocam mudanças no estilo de vida e no envolvimento social como temor de rejeição ou a reação de outros. (NARCHI \& GUTIÉRREZ, 1997)

A aparência, o visual nos é repassado em nossa cultura como o belo, o estar bonito, elegante, sentir-se bem. É percebida nas mulheres uma preocupação em relação a sua imagem onde algumas delas deixam de se olhar no espelho; de se tocar, ficando muitas vezes com vergonha das outras pessoas. (FUNGHETTO et al, 2003)

A paciente tem a preocupação maior com a mutilação que com a doença, pois a morte para ela é fato consumado para os portadores do câncer. (FIALHO, 1993)

Algumas pacientes vivenciam perdas de companheiros ou perdas de amigos após a doença o que reforça o sentimento do estigma social. (CALIRI et al, 1998)

Segundo GRAUDENZ (1981), apud GRACIOLI et al. 2003, p. 145), "quando este símbolo corpóreo, intensamente carregado de narcisismo é mutilado, a auto-imagem pode danificar a mulher e estar presente sentimentos de desamparo, de repulsão e de angústia". Este mesmo autor ainda afirma, distúrbios da identidade feminina em que, além da perda da mama a mulher tem que lidar com a calvície parcial ou total, além de irregulares da menstruação, entre outros.

\section{EXPECTATIVAS}

\section{Uma nova visão}

A confirmação do diagnóstico de conviver com o câncer trouxeram profundas mudanças em suas vidas e na maneira de ver o mundo. As formas de enfrentamento da vida, após o câncer se expressaram nas expectativas voltadas para cura e a necessidade de apego religioso.

Para mulher com diagnóstico de câncer de mama, estabelecer novos propósitos na vida é o resultado dos ajustamentos psicossociais trazidos pelo diagnóstico. Ao aprender e reconhecer que pode alterar sua vida diária, a mulher integra o seu novo ser de forma produtiva e saudável. A esperança de voltar à normalidade é um importante fator facilitador do tratamento. (BERGAMASCO \& ÂNGELO, 2001).

Durante todo o enfrentar da doença e seu tratamento as mulheres procuram ver as coisas do lado positivo para poderem ter esperanças. Após o diagnóstico, as mulheres relatam sentir-se diferentes, haviam mudado em alguns aspectos, seja na sua maneira de ser, seja na maneira de ver a vida. (CALIRI et al, 1998).
Após o tratamento do câncer de mama, algumas iniciativas são tomadas, retorno ao trabalho; dedicação a obras sociais; maior dedicação e proximidade da família e de si própria; maior dedicação e envolvimento no centro espírita; atuação como voluntária num grupo de apoio a mastectomizadas e participação no grupo de pós mastectomizadas com a realização dos encontros de Yoga e Musicoterapia. (BACKES, 1997).

As mulheres ao estabelecerem um vínculo de confiança com um Ser Superior fazem desse vinculo um fio condutor que lhes proporcionam a segurança, a tranqüilidade e força para superarem a dor e o desconforto, aceitando-os e conformando-se com eles. A perspectiva de vida está direcionada na presença e na sintonia com Deus. (BITTENCOURT \& CADETE, 2002b)

SILVA \& MAMEDE (1998, p.134) afirmam que "a manifestação da esperança é um ponto forte para assegurar à mulher uma imagem positiva das mudanças ocorridas no seu corpo. Ajudar outra pessoa, partilhar experiências e problemas ao diagnóstico, ao tratamento, e até mesmo a problemas de outra natureza, são elementos considerados essenciais para o crescimento e/ou transformação do comportamento".

\section{CONSIDERAÇÕES FINAIS}

Acredito ser oportuno a identificação dos sentimentos/comportamentos e expectativas da mulher frente ao câncer de mama, visto que, quando uma pessoa descobre que é portadora de uma neoplasia maligna ela passará por situações conflitantes em que se faz necessário buscar ser compreendida e que não se sinta e esteja sozinha.

Foi possível identificar que o sofrimento estava presente na experiência, pois estava relacionado na percepção que a mulher tem de si como portadora de câncer e com a reformulação e construção de uma nova identidade para mulher com diagnóstico de câncer de mama.

Apreciação feita através dos dados evidenciados nos artigos analisados deixa nítido o impacto causado pelo câncer de mama no cotidiano das mulheres, evidenciando a possibilidade de desenvolvimento de ações efetivas direcionadas à assistência integral.

Viver com uma doença estigamatizante, conviver com sentimentos negativos e enfrentar o tratamento e suas conseqüências significaram para essas mulheres estarem constantemente inseguras e com incertezas, momento este em que se torna importante a participação do profissional de saúde, especialmente das enfermeiras, por percebem que tal diagnóstico traz um significado em suas vidas e suas formas de enfrentamento, com o fim de ajudá-las a explorar seus sentimentos, expectativas e estratégias de ajustamento.

TRAVELBEE (1979), apud GRACIOLI et al (2003) parte do pressuposto que o sofrimento é uma experiência comum que toda pessoa vivencia em algum momento de sua vida, particularmente em torno da doença. Essa experiência é dividida desde o primeiro encontro até a fase do rapport. 
Os resultados do trabalho puderam evidenciar a oportunidade para que a assistência de enfermagem inclua medidas para prevenir ou minimizar a angustia referida pela mulher diante do diagnóstico. Entender as questões referentes ao ajustamento psicossocial permite avaliar e assistir a mulher mais apropriadamente.

\section{REFERÊNCIAS BIBLIOGRÁFICAS}

ALMEIDA, A.M.; MAMEDE, M.M.; PANOBIANCO, M.S. Construindo o significado da recorrência da doença: a experiência de mulheres com câncer de mama. Rev. Latino-Am. Enfermagem, v. 9, n. 5, p. 6369. 2001.

BACKES, V.M.S. A experiência do processo de viver e adoecer para mulheres pós-mastectomizadas. Texto Contexto Enferm., v. 6, n.1, p. 305-316.1997.

BERGAMASCO, R.B.; ANGELO, M. O sofrimento de descobrir-se com câncer de mama: como o diagnóstico é experienciado pela mulher. Rev. Bras. Cancerol., , v. 47, n. 3, p. 277-282. 2001.

BITTENCOURT, J.F.V.; CADETE, M.M.M. Vivências da mulher a ser mastectomizada: esclarecimentos e orientações. Rev. Bras. Enferm., v. 55, n. 4, p.420-423. 2002a.

BITTENCOURT, J.F.V.; CADETE, M.M.M. Deus é a presença incondicional à mulher com possibilidade de vir a ser mastectomizada. Rev. Min. Enf., v. 6, n. 1/2, p. 73-77. 2002b.

BRASIL. Ministério da Saúde. Instituto Nacional do Câncer. Câncer de Mama. Disponível em: http://www.saude.gov.br [Acesso em 29 jan. 2004].

BRASIL. Ministério da Saúde. Instituto Nacional do Câncer. Falando sobre o câncer de mama. Rio de Janeiro: INCA, 2003. CD-ROM.

CALIRI, M.H.L.; ALMEIDA, A. M.; SILVA, C.A. Câncer de mama: a experiência de um grupo de mulheres. Rev. Bras. de Cancerol, v. 44, n. 3, p. 239-247.1998.

CORBELLINI, V.L. Câncer de mama: encontro solitário com o temor do desconhecido. Rev. Gaúcha Enferm., v. 22, n. 1, p. 42-68. 2001.

FERNANDES, A.F.C.; MAMEDE,M.V. O surgimento do câncer de mama na visão de um grupo de mulheres mastectomizadas. Texto Contexto Enferm., v. 13, n. 1, p. 35-40. 2004.

FERREIRA, Aurélio Buarque de Holanda. Minidicionário da Língua Portuguesa. 3ed. Rio de Janeiro: Nova Fronteira. 1993.

FIALHO, A.V.; SILVA, R.M. Mastectomia e suas repercussões. Rev. Bras. Enferm., v. 46, n. 3/4, p. 266270.1993.

FUNGHETTO, S.S.; TERRA, M.G.; WOLFF, L.R. Mulher portadora de câncer de mama: percepção sobre a doença, família e sociedade. Rev. Bras. Enferm, v. 56, n. 5, p. 528-532. 2003.

GALVÃO, C M; SAWADA, N. O.; TREVIZAN, M A. Revisão Sistemática: recurso que proporciona a incorporação das evidências na prática da enfermagem. Rev. Latino-Am. Enferm.,v. 12, n. 3, p. 549-556. 2004.

GIL, A. C. Métodos e técnicas da pesquisa social. $5^{\mathrm{a}}$ ed. São Paulo: Atlas, 1999.

GRACIOLI, Michelle Araújo da Silva; LEOPARDI, Maria Tereza; GONZALES, Rosa Maria Bracini. Assistência de Enfermagem a Mulheres Mastectomizadas com Bases nos Conceitos e Pressupostos de Joyce Travelbee. Separata de: COSTENARO, Regina G. Satini. Cuidando em Enfermagem: da Teoria à Prática. Rio Grande do Sul: Unifra, 2003. cap. 04, p.121-165

NARCHI, N.Z.; GUTIÉRREZ, M.G.R. Diagnóstico de enfermagem em mulheres com câncer de mama submetidas à quimioterapia antineoplásica. Acta Paul. Enf., v. 10, n. 1, p. 10-17, 1997.

NEGRINI, Marize Regina. Relacionamento Terapêutico Enfermeiro-Paciente Junto a Mulheres Mastectomizadas, 1994. 96f. Dissertação (Mestrado em Enfermagem) Escola de Enfermagem de Ribeirão Preto, Universidade de São Paulo.

POLIT, Denise F; HUNGLER, Bernadette $P$. Fundamentos de Pesquisa em Enfermagem. 3ed. Porto Alegre: Artes Médicas. 1995

SILVA, R. M.; MAMEDE, M. V. Conviver com a Mastectomia. Fortaleza: Departamento de Enfermagem da Universidade Federal Ceará.1998.

\footnotetext{
${ }^{1}$ Graduanda da Escola de Enfermagem Aurora de Afonso Costa da Universidade Federal Fluminense.

${ }^{2}$ Enfermeira. Profa. Dra. da Escola de Enfermagem Aurora de Afonso Costa.E-mail: soniamarafsimoes@aol.comEnd: Av. Meriti n. 942 apto. 101 - Vila da Penha - RJ. CEP:21220204
} 